\title{
La inmersión concepto-lineal como dinamizadora del proceso de aprendizaje de formadores con una visión global de los contenidos matemáticos
}

The linear-concept inmersion like as dynamic of the teacher's learning process in mathematics.

Jorge Robinson Evilla

Universidad del Norte-Universidad del Atántico. jrobinso@uninorte.edu.co

\section{Resumen}

Se toma una definición como eje y se analizan dos tipos de inmersiones. La primera es por medio de definiciones que se construyen a partir de la definición eje y la segunda es variando los espacios sobre los que se ha construido la definición. En este trabajo se muestra la inmersión concepto lineal para la definición de límite.

\section{Palabras clave}

Concepto, inmersión, límite, derivada.

\section{Abstrac}

I take a definition like axis and analyze two kinds of inmersions. The first is for the definitions that use the axis-definition and the other is to changing the spaces where the definition is included.

\section{Keywords}

Concept, inmersion, limit, derivative.

Se entiende por inmersión concepto-lineal al desarrollo temático con un concepto que funciona como eje y que permanece constante. En nuestro caso estamos interesados en analizar dos tipos de inmersiones para un concepto- eje. La inmersión por definiciones y la inmersión por espacios.

La primera de las inmersiones consiste en tomar una definición y luego enunciar definiciones que la utilicen. Esto tiene como objetivo permitir analizar la definición contrastada con las otras. En este trabajo se presentará la definición de límite de una función, como definición eje, junto a definiciones secundarias de continuidad, derivada e integral definida, que no podrían existir sin la definición de límite. Se intenta que el formador, además de preocuparse por la definición en sí, sea capaz de reconocerla en otras definiciones mas complejas. En el caso particular de la derivada y la integral, es necesario verlas como límites para evitar la simple manipulación y el simple cálculo, así, aparte de estructurar mejor el concepto de límite al verlo actuando como derivada o integral, también entendemos mejor las otras definiciones. Esto quiere contribuir a evitar la inconexidad en el estudio de las matemáticas. Las definiciones aisladas tienden a debilitarse y a generar inquietudes que aunque interesantes no aportan en el crecimiento disciplinar. El conocimiento del formador debe abarcar las relaciones entre definiciones, esto le permitirá construir un discurso coherente y ordenado. Una visión de las definiciones que permita ir de la esencial a la mas compleja nos da daridad y coherencia. Si se logra analizar con detalle las definiciones podremos terminar viendo la continuidad, la derivada y la integral como versiones de mayor complejidad del concepto de límite. 
La segunda de las inmersiones consiste en analizar la definición y variar los espacios donde se analiza. En nuestro caso particular, de la definición de límite de una función, hacemos variar los espacios donde está definida la función. Iniciamos con funciones definidas sobre espacios topológicos, luego espacios métricos, después espacios normados, y por último espacios vectoriales. En esta parte las posibilidades de análisis son mayores. Podríamos induso pensar que son inagotables, pero justo este tipo de razonamientos permite ampliar la visión, fortalecer el concepto y construir un discurso pedagógico con un gran impacto positivo en el crecimiento intelectual de los estudiantes, objetivo final del trabajo con formadores. Se intenta aprovechar el desarrollo conceptual de los formadores y poner a prueba un modelo de aprendizaje que resalte el significado a partir de propiedades y comportamientos en diferentes espacios y sentidos. Un aprendizaje basado en lo global que por medio de los conceptos centrales genere conocimiento útil y contextualizado que permita aplicaciones y modelado.

La siguiente es la propuesta de la inmersión concepto-lineal por definiciones, del concepto de límite de una función de valor real y variable real. La primera parte está conformada por las definiciones de límite, continuidad, derivada e integral definida. La segunda parte es un grupo de preguntas que intentan cumplir el objetivo de estructuración del concepto de límite a partir de definiciones de las que forma parte.

\section{Inmersión concepto-lineal del límite de una función por definiciones.}

Parte I. Definiciones a analizar:

1. Definición de límite de una función.

El simbolismo: $\lim _{x \rightarrow p} f(x)=A$ significa que para todo $\varepsilon>0$, existe $\delta>0$, tal que: $|f(x)-A|<\varepsilon$ siempre que $0<\|x-p\|<\delta$.

2 Definición de continuidad.

Se dice que una función $f$ es continua en un punto p, si: a) $f$ está definida en p, y b) $\varliminf_{x \rightarrow p} f(x)=f(p)$.

3. Definición de derivada.

La derivada de $f^{\prime \prime}(x)$ está definida por la igualdad: $f^{\prime}(x)=\lim _{h \rightarrow 0} \frac{f(x+h)-f(x))}{h}$ con tal que este límite exista.

4. Definición de Integral Definida.

Sea $F$ una función ayyo dominio es el intervalo cerrado $[a, b]$, sea $P_{n}$ una partición de [a, b] determinada por el conjunto $\left\{x_{0}, x_{1}, \ldots, x_{n}\right\}$ y arya norma es $N_{p}$ y sea $T_{n}=\left\{t_{1}, t_{2}, \ldots, t_{n}\right\}$ un aumento de la partición $P_{n}$. Sea: $\Delta x_{i}=x_{i}-x_{i-1}$ para $i=1,2,3, . . n$. Definimos la suma de Riemann de $F$ en el intervalo $[a, b]$ para la partición $\mathbf{P}_{n}$ y el aumento $\mathbf{T}_{n}$ así: $\mathbf{S}\left(\mathbf{F} ; \mathbf{P}_{\mathbf{n}} \mathbf{T}_{\mathbf{n}}\right)=\sum_{i=1}^{n j} F\left(\mathbf{t}_{\mathbf{i}}\right) \Delta \mathbf{x}_{\mathbf{i}}$. Si existe un número I tal que para todo $\varepsilon \geqslant 0$ exista un $\delta \geqslant 0$ tal que: IS(F; $\left.\mathbf{P}_{\mathbf{n}} \mathbf{T}_{\mathbf{n}}\right)-\mathrm{Il}<\varepsilon$ para todas las particiones $P_{n} y$ todos los aumentos $T_{n}$ con $N_{p}<\delta$, a este número I le llamamos la integral definida en el sentido Riemann de $F$ en $[a, b]$.

Parte II. Preguntas a responder:

1. ¿Qué propiedades de la derivada provienen directamente del hecho de ser un límite? 
2. ¿Qué propiedades de la integral provienen directamente del hecho de ser un límite?

3. ¿Qué propiedades tienen en común la derivada y la integral?

4. ¿Qué propiedades se aumplen en la derivada que no se cumplan en la integral?

5. ¿Qué de la definición de continuidad se necesita para la definición de derivada?

6. ¿Por qué si el límite del producto de dos funciones es igual al producto de los límites, auando estos existen, se necesita la regla del producto para la derivación?

7. ¿Puede inferirse de la definición misma de derivada la continuidad como una consecuencia?

Esta actividad tiene un gran potencial para la formalización del concepto de límite y al mismo tiempo permite analizar en conjunto, continuidad, derivada e integral. Este ejemplo nos muestra que es posible utilizar todo el conocimiento que ya tienen los formadores, al tiempo que se les propone enfocar el nuevo estudio de la temáticas desde un enfoque globalizador y formal. El hecho de que las preguntas no tengan respuestas exactas, también genera discusión y uso de lo aprendido, que en el caso de formadores es bastante y no se justifica desaprovecharlo partiendo de cero. Al tiempo que se recuerda lo elemental, se fortalece lo esencial de las definiciones y el formador queda en un nivel superior, en lo que a abstracción se refiere, de sus estudiantes.

\section{Inmersión concepto-lineal del límite de una función por cambio de espacios}

Parte I. Definiciones a analizar:

1. Definición de Espacio Topológico.

Sea $X$ un conjunto aualquiera. Se llama topología en $X$ a todo sistema $T$ de subconjuntos de $X$ que verifica: i) $X \in T, \emptyset \in T$. ii) La unión de un número aualquiera de elementos de $T$ está en $T$ y la intersección de un número finito de elementos de $T$ pertenece a $T$. Al conjunto $X$ junto con $T$, es decir, al par $(X, T)$ se le llama espacio topológico.

2. Definición sucesión convergente en un espacio topológico.

La sucesión $\left\{x_{n}\right\}$ de puntos de $X$ converge al punto $x$, si toda vecindad del punto $x$ contiene todos los puntos de esta sucesión, excepto posiblemente un número finito de ellos.

3. Definición de Espacio Métrico.

Un espacio métrico es un conjunto $M$, no vacío, dotado de una función $d$ de $M \times M$ en $R$, que satisface, para aualesquiera puntos $x, y, z$ de $M$ :
a. $d(x, x)=0$ si y solo si $x=y$.
b. $d(x, y)>0$, si $x=y$.
c. $d(x, y)=d(y, x)$
d. $d(x, y) \leq d(x, z)+d(z, y)$

4. Definición de límite en un espacio métrico.

Sean $\left(S, d_{s}\right)$ y $\left(T, d_{T}\right)$ dos espacios métricos. f:S $\rightarrow T$ es una función de $S$ en $T$. Si p es un punto de acumulación de $\mathbf{S}$ y si $\mathbf{b} \in \mathbf{T}$, la notación: $\lim _{x-p} f(x)=b$, significa que para todo $\varepsilon>0$, existe $\delta>0$, tal que: $d_{\mathrm{T}}(f(x), b)<\epsilon$ siempre que $x \in S, x=p$ y $d_{s}(x, p)<\delta$.

5. Definición de norma. 
Una norma sobre un espacio vectorial $\mathbf{X}$ es una función de valor real sobre $\mathbf{X}$, ayyo valor en $x$ se denota $\|x\|$ y que satisface: i) $\|x\| \geq 0$, ii) $\|x\|=0 \leftrightarrow x=0$, iii) $\|\alpha x\|=\|\alpha\|\|x\|$, iv) $\|x+y\| \leq\|x\|+\|y\|$, donde $x$ e $y$ son elementos de $X \mathbf{y} \alpha$ es aralquier escalar.

6. Definición de sucesión convergente en un espacio nomado.

Una sucesión $\left\{x_{n}\right\}$ en un espacio normado $\mathbf{X}$ es convergente si $\mathbf{X}$ contiene un $x$ tal que: $\lim _{n \rightarrow \infty}\left\|x_{m}-x\right\|=0$. A $x$ lo llamamos el límite de $\left\{x_{n}\right\}$.

Parte II. Preguntas a responder:

1. Construya un espacio topológico a partir de una métrica.

2. Construya un espacio normado a través de una métrica.

3. De un ejemplo de un espacio topológico que no sea una métrica.

4. ¿Qué propiedades tiene el límite en un espacio normado que no necesariamente tiene en un espacio topológico?

5. ¿Qué relación tiene la definición de límite de una función de valor real y variable real con la definición de límite en espacios métricos?

6. ¿Qué relación tiene la definición de límite de una función de valor real y variable real con la definición de límite en espacios normados?

\section{Comentarios finales}

Estas dos actividades, presentan un enfoque mas analítico y reflexivo del trabajo con formadores. La daridad conceptual se hace impresaindible en los formadores y son necesarias actividades enfocadas al aumento del conocimiento de las relaciones y dependencias entre los conceptos básicos. El concepto de límite estará presente en la mayoría de las aplicaciones y de los contenidos de la enseñanza de la matemática, ya sea directamente o haciendo parte de una definición que se está utilizando. La construcción del concepto de límite se realiza a través del análisis de sus relaciones con otras definiciones y de sus variaciones al cambiar elementos de ella. Los formadores deben estar bien preparados en los conceptos esenciales de forma que sugieran caminos que permitan que los estudiantes avancen en el conocimiento y la abstracción matemática.

\section{Bibliografia}

Apostol, T. (1972). Calculus. Barcelona: Editorial Reverté, S. A.

Kolmogorov, A y Fomin. S. (1972) . Elementos de la teoría de funciones y del análisis funcional. Moscú: editorial Mir. 